\title{
NOTES
}

\section{Electric and Magnetic Orientation of a Thermotropic-Liquid Crystalline Polypeptide}

\author{
Eisaku IIzUKa, Takeshi KIRIKI, and Koji ABE \\ Department of Functional Polymer Science, Faculty of Textile Science and \\ Technology, Shinshu University, Ueda, Nagano 386, Japan \\ (Received January 18, 1991) \\ KEY WORDS Polypeptide / Thermotropic Liquid Crystal / Electric \\ Orientation / Magnetic Orientation /
}

In a preceding paper, ${ }^{1}$ one of the authors (E.I.) reported that an $\alpha$-helical poly (L-glutamate) ester with alkyl side chains having 6 carbons containing 1 benzene group at the terminal position exhibited spontaneous orientation in the direction of the axis of a X-ray glass capillary when in a thermotropic-liquid crystalline state at $160^{\circ} \mathrm{C}$. Arrangement of polymer helices was hexagonal in this state and turned to rectangular when cooled to room temperature where the specimen was in a crystalline state. In this paper, we wish to report further that the thermotropic-liquid crystal of this polypeptide oriented both in an electric and a magnetic fields.

The abbreviation for this specimen is $\mathrm{R}^{\prime \prime} 6$ according to the preceding paper; the structural formula of $R^{\prime \prime} 6$ is shown below. The degree of

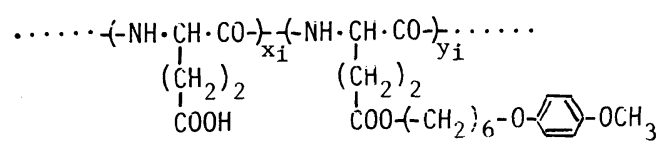

polymerization of $\mathrm{R}^{\prime \prime} 6, \Sigma X_{i}+\Sigma y_{i}$, is 940 , and degree of substitution being $98 \%$. This specimen undergoes the crystal-to-liquid crystal transition at about $27^{\circ} \mathrm{C}$. The specimen was put in a cubic quartz cell $10 \mathrm{~mm}^{2}$ inside, heated at $160^{\circ} \mathrm{C}$ in an oil bath for 10 hours under an electiric field of $400 \mathrm{Vcm}^{-1}$ between 2 platinum electrodes $7.5 \mathrm{~mm}$ apart inside the cell, and cooled naturally to room temperature to assure the full growth of a crystalline structure. The specimen was also heated at $120^{\circ} \mathrm{C}$ by a nichrome-wire heater which was put round the cubic cell for 1 week under a magnetic field of 6,000 gauss between the poles of the permanent magnet described elsewhere. ${ }^{2}$

Figure 1 shows X-ray diffraction photographs of specimens so treated. The spacing corresponding to the innermost diffraction is $22.6 \AA$ in the two cases. This reflection originates from the (200) lattice planes of the rectangular unit cell. ${ }^{1}$ It appears on the equator in the electrically oriented specimen and on the meridian in the magnetically oriented specimen. The direction of the external field was vertical and this experimental evidence clearly indicates that $R^{\prime \prime} 6$ is oriented both in electric and magnetic fields and that arrangement of the polymer helices is parallel to the applied field when treated in electric field and perpendicular to the applied field when treated in magnetic field. The degree of orientation is rather low in both cases.

The peptide group, $-\mathrm{NH} \cdot \mathrm{CO}-$, of a polypeptide has a permanent electiric dipole moment of about 6 Debye. When in the $\alpha$ helical conformation, all the peptide groups roughly orient in the same direction. Therefore, the polymer molecule has a resultant large dipole moment in the direction of the polymer helix. In the case of poly ( $\gamma$-benzyl-L-glutamate) 

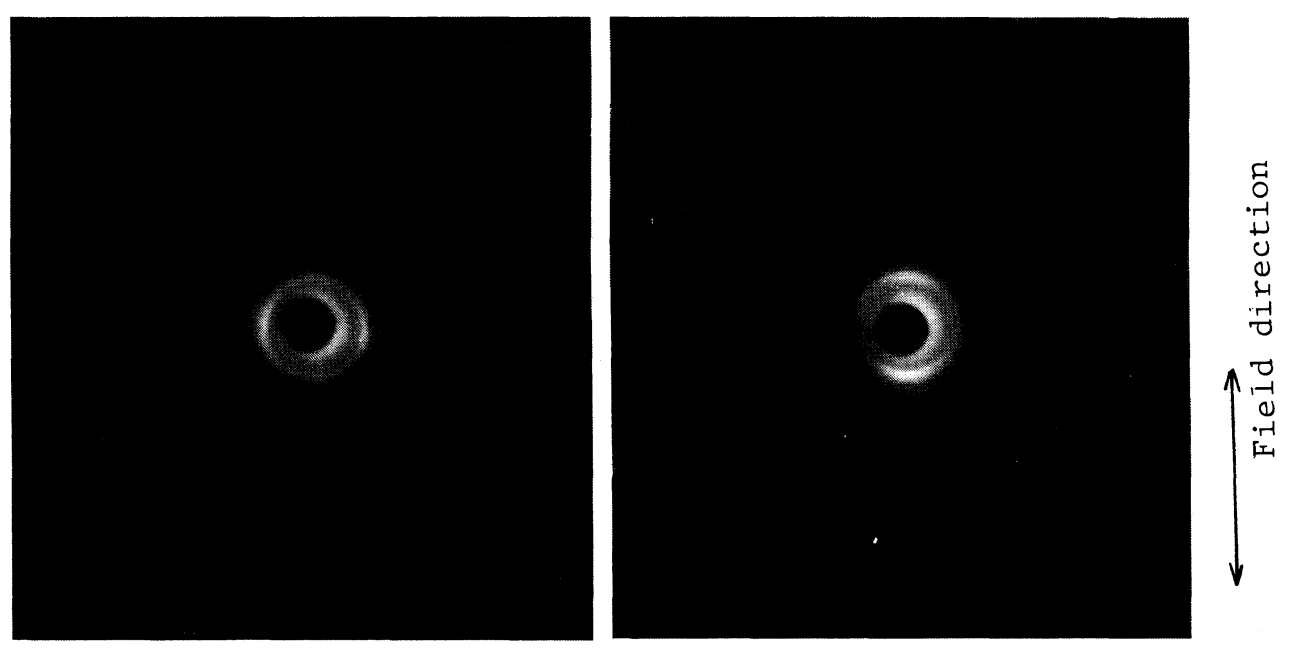

Figure 1. X-Ray diffraction photographs of an electrically oriented (left) and a magnetically oriented (right) $\mathrm{R}^{\prime \prime} 6$. Cu- $K_{\alpha}$ radiation, $D=9.3 \mathrm{~cm} ; D$ is the distance from the sample to the photographic film. The direction of the applied field, vertical.

(PBLG), the magnitude of the dipole moment per residue turns out to be 3.5 Debye because of the contribution of dipoles originated in the side chains which point the reverse direction. ${ }^{3}$ This polypeptide orients very well in the direction of an applied electric field as low as $84 \mathrm{~V} / \mathrm{cm}$ when dissolved in dibromomethane and forms liquid cystals. ${ }^{4}$ This orientation is brought about as the result of cooperative behaviour of th polymer helices in lyotropicliquid crystalline state. Since the pendant alkyl side chains of $\mathrm{R}^{\prime \prime} 6$ behave as a solvent in a similar manner as in the lyotropic-liquid crystals of PBLG when in thermotropic-liquid crystalline state, ${ }^{2}$ it would be very natural that $\mathrm{R}^{\prime \prime} 6$ exhibits orientation in the direction of the applied electric field.

Polypeptides such as PBLG and poly $(\gamma-$ ethyl-L-glutamate) (PELG) are known to orient in the direction of an applied magnetic field when in lyotropic-liquid crystalline state. ${ }^{5-7}$ According to Suzuki and Wakabayashi, ${ }^{8}$ the atoms constituting the peptide group are in a plane because of the delocalization of $\pi$ electrons which are on both sides of this plane. If a magnetic field is applied to an $\alpha$-helical polypeptide molecule so that it is perpen- dicular to the planes of the peptide groups, a demagnetizing field, or diamagnetic dipoles in another expression, is (or are) induced by the ring current of the $\pi$ electrons parallel to the applied magnetic field. This makes the situation of the polymer molecule unstable. Since the planes of the peptide groups are roughly parallel to the polymer axis, the polymer molecule is forced to become parallel to the applied magnetic field. Cooperative behavior of the polymer helices in liquid-crystalline state plays an important role as in the case of electric orientation.

According to Tsuboi, ${ }^{9} 2$ transition moments, the Phenyl $A_{1}$ and Phenyl $B_{1}$, of PBLG are both in the plane of benzene ring and are perpendicular with each other. The direction of the Phenyl $A_{1}$ is at $46^{\circ}$ and that of the Phenyl $B_{1}$ is at $61^{\circ}$ both with respect to the helical polymer axis. This suggests that the planes of the benzene rings which are distributed around the polymer axis at an equal probability roughly make the magic angle of $54^{\circ} 44^{\prime}$ with the polymer axis. The benzene ring induces a diamagnetic dipole of which the direction is perpendicular to its plane in a similar manner as the peptide group does. Therefore, the angle 
that the direction of this dipole makes with the polymer axis is close to the magic angle, suggesting that contribution of the benzene groups of PBLG to the magnetic orientation is small. However, the observed anisotropy of magnetic susceptibility, $\chi_{\|}-\chi_{\perp}$, is about 2 times larger in PBLG than in PELG which lacks benzene groups; it is $4.52 \times 10^{-8} \mathrm{emu} / \mathrm{g}$ and $(3.6 \pm 0.2) \times 10^{-8} \mathrm{emu} / \mathrm{g}$ for $\mathrm{PBLG},{ }^{6,10}$ and $(1.64 \pm 0.16)^{-8} \mathrm{emu} / \mathrm{g}$ for PELG. ${ }^{11}$ This suggests that the tilt angle of the benzene plane is somewhat smaller than the magic angle and that the anisotropy is considerably larger in benzene group than in peptide group.

The intensity of the magnetization of a rod, $I$, is defined as the magnetic charge induced per unit area of the terminal plane of the rod, and is equal to the magnetic moment per unit volume of the rod. Since $I=\chi H$ where $\chi$ is the magnetic susceptibility and $H$ is the magnetic field inside the magnetic substance, the anisotropy of the magnetic susceptibility turns out to be the magnetic moment per unit volume and per unit field strength. Dividing the observed anisotropy of PELG $\left(1.64 \times 10^{-8} \mathrm{emu} /\right.$ g) by the number of peptide group in 1 gram of PELG, the induced magnetic moment due to the peptide group was estimated to be $4.6 \times$ $10^{-30} \mathrm{emu} /$ gauss.

The induced magnetic moment (diamagnetic), $\mu$, of a benzene group was given by Pople, ${ }^{12}$ in the form: $\mu=3 e^{2} H S / 2 \pi m c, S$ is the area of the benzene plane and is roughly to $\pi a^{2}$ where $a$ is the $\mathrm{C}-\mathrm{C}$ distance; $e, m$ and $c$ have their usual meanings and Gaussian system of units being used. The value of $\mu$ was then calculated at $3.5 \times 10^{-18} \mathrm{emu} /$ gauss. This value is far larger than that of the peptide group more than expected. The experimental evidence that the polymer helices of $\mathrm{R}^{\prime \prime} 6$ oriented in the direction

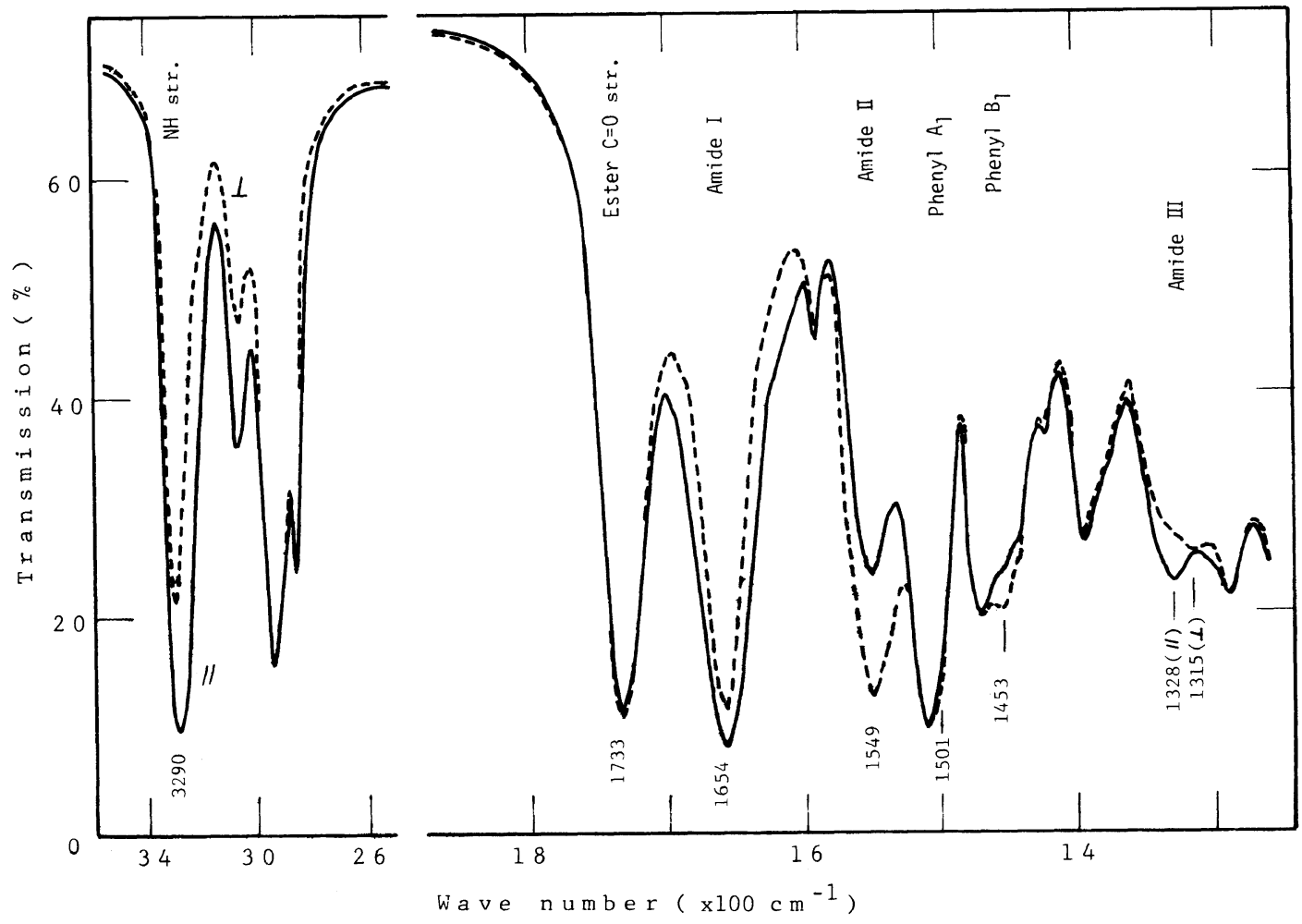

Figure 2. Infrared absorption spectra of an oriented $R^{\prime \prime} 6$. Reference lines for calculating the dichroic ratio of the Phenyl $\mathbf{B}_{1}$ mode are shown on the absorption curves. 
perpendicular to the applied magnetic field unlike those of PBLG suggests that the planes of the benzene groups of $\mathrm{R}^{\prime \prime} 6$ are distributed perpendicular rather than parallel to the polymer axis.

A small amount of $R^{\prime \prime} 6$ was put on a calcium fluoride plate, and was spread in one direction with a spatula and a forefinger cushion to make an oriented polymer film. Figure 2 shows infrared absorption spectra of $\mathrm{R}^{\prime \prime} 6$ so prepared. The dichroic ratio was calculated for a couple of transition modes, namely the NH stretching vibration mode at $3290 \mathrm{~cm}^{-1}$ and the Phenyl $B_{1}$ mode at $1453 \mathrm{~cm}^{-1}$. The orientation factor, $\Phi$, of an oriented sample can be calculated using the following equation, ${ }^{4}$

$$
\begin{aligned}
\Phi & =\frac{1}{2}\left(3<\cos ^{2} \theta>_{\mathrm{AV}}-1\right) \\
& =\frac{\left(D_{\|} / D_{\perp}\right)-1}{\left(D_{\|} / D_{\perp}\right)+2} \cdot \frac{2}{2-3 \sin ^{2} \gamma}
\end{aligned}
$$

where $\theta$ is the angle that the polymer axis makes with the direction of orientation, $D_{\|} / D_{\perp}$ is the dichroic ratio of any transition moment, and $\gamma$ is the tilt angle that the transition moment in question makes with the polymer axis. Using Tsuboi's result ${ }^{9}$ that $\gamma=28^{\circ}$ for the $\mathrm{NH}$ stretching vibration mode, the $\Phi$-value of the oriented $\mathrm{R}^{\prime \prime} 6$ was calculated. The tilt angle of the Phenyl $B_{1}$ mode, which shows the perpendicular dichroism, was then estimated with the thus calculated $\Phi$-value and observed dichroic ratio of this mode.

The mean values of 4 samples tested were 0.36 for the orientation factor, and $64^{\circ}$ for the tilt angle of the Phenyl $\mathrm{B}_{1}$ transition moment. Since absorptions due to neighboring 2 transition modes fall on the absorption due to the Phenyl $B_{1}$ mode, the true dichroic ratio of this mode is undoutedly smaller than the mean observed value (0.81) and the true tilt angle of the Phenyl $B_{1}$ transition moment being lager than $64^{\circ}$, accordingly. Although the absorption due to the Phenyl $A_{1}$ mode is recognized only as a weak shoulder because of a neighboring strong absorption centered at $1501 \mathrm{~cm}^{-1}$, this transition mode exhibits the sign of the perpendicular dichroism. Thus, both 2 Phenyl transition moments, which are in the plane of the benzene ring and perpendicular with each other, show the perpendicular dichroism. This experimental evidence suggests that the planes of the benzene groups of $R^{\prime \prime} 6$ are perpendicular rather than parallel to the polymer axis. This agrees with the idea concerning the arrangement of the side-chain benzene groups of $\mathrm{R}^{\prime \prime} 6$.

It should be noticed, however, that the oriented films were prepared in liquid crystalline state where the alkyl side-chains of the polymer helices were melted though not completely as in free alkanes. Therefore, arrangement of the planes of the benzene rings which are combined to the terminal position of the alkyl chains might have fallen into disorder considerably. This would explain why the polymer helices did not show good orientation in a magnetic field.

Acknowledgment. We wish to thank Drs. H. Shirai and K. Hanabusa of our Faculty for the donation of the polypeptide specimen used in this study.

\section{REFERENCES}

1. E. Iizuka and S. Iida, Polym. J., 22, 628 (1990).

2. E. lizuka and Y. Go, J. Phys. Soc. Jpn., 31, 1205 (1971).

3. A. Wada, J. Chem. Phys., 30, 328 (1959).

4. E. Iizuka, Biochim. Biophys. Acta, 243, 1 (1971).

5. S. Sobajima, J. Phys. Soc. Jpn., 23, 1070 (1967).

6. R. W. Duke and D. B. DuPre, J. Chem. Phys., 60, 2759 (1974).

7. E. Iizuka, Polym. J., 5, 62 (1973).

8. S. Suzuki and K. Wakabayashi, KEK Reports, 83-23 (1984).

9. M. Tsuboi, J. Polym. Sci., 59, 139 (1962).

10. K. Tohyama and E. Iizuka, J. Phys. Soc. Jpn., 37, 1172 (1974).

11. K. Tohyama and N. Miyata, J. Phys. Soc. Jpn., 34, 1699 (1973).

12. J. A. Pople, J. Chem. Phys., 24, 1111 (1956). 